\title{
The Characteristics of Cognitive Impairment in ALS Patients Depend on the Lateralization of Motor Damage
}

\author{
Umberto Manera ${ }^{1, \dagger}{ }^{\dagger}$ Laura Peotta ${ }^{1, \dagger}{ }^{\dagger}$ Barbara Iazzolino ${ }^{1}$, Antonio Canosa ${ }^{1,2}$, Rosario Vasta ${ }^{1}$, \\ Francesca Palumbo ${ }^{1}$, Maria Claudia Torrieri ${ }^{1}$, Luca Solero ${ }^{1}$, Margherita Daviddi ${ }^{1}$, \\ Maurizio Grassano ${ }^{1}$, Cristina Moglia ${ }^{1,2}\left(\mathbb{D}\right.$, Marco Pagani ${ }^{3,4}$, Adriano Chiò 1,2,3, $\ddagger$ and \\ Marco Cavallo ${ }^{5, *, \ddagger(D)}$ \\ 1 ALS Centre, Department of Neuroscience "Rita Levi Montalcini”, University of Torino, 10126 Turin, Italy; \\ umberto.manera@gmail.com (U.M.); lapeo92@gmail.com (L.P.); barbara.iazzolino@gmail.com (B.I.); \\ antoniocanosa85@gmail.com (A.C.); sarovasta@gmail.com (R.V.); fra-palumbo@libero.it (F.P.); \\ mariaclaudia.torrieri@gmail.com (M.C.T.); lucasolero91@gmail.com (L.S.); \\ margherita.daviddi@gmail.com (M.D.); grassano.maurizio@gmail.com (M.G.); \\ cristina.moglia@unito.it (C.M.); adriano.chio@unito.it (A.C.) \\ 2 SC Neurologia 1U, AOU Città della Salute e della Scienza of Torino, 10126 Turin, Italy \\ 3 Institute of Cognitive Sciences and Technology, CNR, 00185 Rome, Italy; marco.pagani@istc.cnr.it \\ 4 Department of Medical Radiation Physics and Nuclear Medicine, Karolinska University Hospital, \\ 17164 Stockholm, Sweden \\ 5 Faculty of Psychology, eCampus University, 22060 Novedrate, Italy \\ * Correspondence: marco.cavallo@uniecampus.it \\ + These authors contributed equally to the paper. \\ $\ddagger$ These authors contributed equally to the paper.
}

Received: 15 July 2020; Accepted: 17 September 2020; Published: 19 September 2020

\begin{abstract}
Background: Cognitive features of patients with amyotrophic lateral sclerosis (ALS) have never been specifically analyzed according to the lateralization of motor impairment. In the present study we investigated the cognitive performances of ALS patients to describe the relationship between motor and cognitive dysfunction, according to site and side of disease onset. (2) Methods: Six-hundred and nine ALS patients underwent a comprehensive neuropsychological evaluation at diagnosis in Turin ALS Centre Tests included-mini-mental state examination (MMSE), frontal assessment battery (FAB), trail-making test A/B (TMT A-B), digit span forward and backward (digit span FW/digit span BW), letter fluency test (FAS), category fluency test (CAT), Rey auditory verbal learning test (RAVLT), Babcock story recall test (BSRT), Rey-Osterrieth complex figure test (ROCFT), Wisconsin card sorting test (WCST), Raven's coloured progressive matrices (CPM47). Cognitive performances of patients, grouped by side and site of onset, were statistically compared using $z$-scores, as appropriate. (3) Results: Bulbar patients and bilateral spinal onset patients $\left(\mathrm{S}_{\mathrm{bil}}\right)$ were generally characterized by lower cognitive performances in most neuropsychological tests, when compared to patients with lateralized onset (right-side spinal onset, $\mathrm{S}_{\mathrm{ri}}$ and left-side spinal onset, $\mathrm{S}_{\mathrm{le}}$ ). Digit span backward and visual memory task (ROCFT) median $z$-scores were significantly higher, reflecting a better cognitive performance, in $S_{\text {ri }}$ patients when compared to bulbar/ $S_{\text {bil }}$ patients, while verbal memory tasks (RAVLT and BRST) resulted in significantly higher scores in $\mathrm{S}_{\text {le }}$ patients. Our results are in keeping with hemispheric functional lateralization of language and visuospatial abilities. (4) Conclusions: In ALS patients, as in other neurodegenerative diseases, we found a direct relationship between lateralized motor and cognitive features.
\end{abstract}


Keywords: amyotrophic lateral sclerosis; neuropsychological evaluation; frontotemporal dementia; hemispheric lateralization; language; visuospatial abilities

\section{Introduction}

Amyotrophic lateral sclerosis (ALS) is a neurodegenerative disorder that primarily involves all voluntary motor functions, through progressive degeneration of upper and lower motor neurons [1]. It is characterized usually by a focal onset in limbs or bulbar muscles, where upper and lower motor signs are maximal [2], spreading through contiguous or network pathways [3]. In limb-onset patients, the site of onset is usually lateralized, especially in patients with upper limb onset, and has been related to limb dominance with a high concordance for side of onset and handedness [4]. Cognitive impairment is a frequent additional feature of ALS [5,6], being mainly related to frontotemporal cortical neuron degeneration, such as motor impairment. There are indications that cognitive function may worsen during disease progression [7], with a correlation to motor dysfunction and clinical stages [8].

Despite this general correlation between motor and cognitive impairment, the patterns followed by neurodegeneration to spread across are not fully understood but seem to maintain a certain degree of lateralization [3]. An area that has been generally neglected in ALS is the notion that some cognitive functions, which can be impaired in the disease, are functionally lateralized, in particular language and visuospatial abilities [9].

The aim of the present study was to analyze the performance of a cohort of ALS patients in different cognitive domains according to the site and side of disease onset, and to identify the presence of specific cognitive patterns related to the lateralization of motor impairment.

\section{Materials and Methods}

\subsection{Data Collection}

The study population included all ALS cases diagnosed between 2010 and 2018 who underwent neuropsychological evaluation at the Turin ALS Center. Site of onset, side of onset, date of onset, sex, hand dominance, and years of formal education were collected using data recorded in the Piemonte and Valle D'Aosta register for ALS (PARALS, [10]). All neuropsychological evaluations were performed at diagnosis (diagnosis-evaluation interval $<3$ months). The battery included a series of tests aimed at evaluating executive function, memory, visuospatial function, and language, selected according to the diagnostic criteria for the behavioral variant of frontotemporal dementia [11] and the amyotrophic lateral sclerosis frontotemporal dementia (ALS-FTD) consensus criteria [12]. Specifically for this study we considered mini-mental state examination (MMSE), Wisconsin card sorting test (WCST), trail-making test A (TMT-A) and B (TMT-B) and (TMT-B-A), digit span forward (digit span FW) and backward (digit span BW), letter (FAS) and category (CAT) fluency test, Rey auditory verbal learning test (RAVLT), Babcock story recall test (BSRT), Rey-Osterrieth complex figure test (ROCFT), Raven's colored progressive matrices (CPM47), and frontal assessment battery (FAB) [13]. The study design was approved by the Ethical Committee of the Azienda Ospedaliero-Universitaria Città della Salute (prot. n. 0036344). Patients signed a written informed consent. Data will be available upon request by interested researchers.

\subsection{Cognitive Categorization}

Patients' cognitive status was classified, according to the revised ALS-FTD Consensus Criteria, [12] into five categories: (1) patients with ALS with a frontotemporal dementia (FTD) syndrome (ALS-FTD); (2) patients with ALS with behavioral impairment (ALSbi); (3) patients with ALS with cognitive impairment (ALSci); and (4) patients with ALS with combined cognitive and behavioral impairment 
(ALScbi), which includes patients who fulfilled criteria for both ALSci and ALSbi. Patients who did not fit into these categories were classified as cognitively normal (ALS-CN).

\subsection{Side of Onset Classification}

Patients' history was carefully evaluated to classify patients by side of onset, considering neurological symptoms such as dysphagia, dysarthria, muscle atrophy, strength and dexterity reduction to attribute disease onset. Muscle cramps and fasciculation were not considered. Patients were classified into four categories: bulbar onset patients, right-side spinal onset patients $\left(\mathrm{S}_{\mathrm{ri}}\right)$, left-side spinal onset patients $\left(\mathrm{S}_{\text {le }}\right)$ and bilateral spinal onset patients $\left(\mathrm{S}_{\mathrm{bil}}\right)$. $\mathrm{S}_{\text {bil }}$ included mainly patients with predominant upper motor neuron predominant phenotype [14], whose symptoms were referred to start in both upper and lower limbs simultaneously.

\subsection{Statistical Analysis}

In order to make proper comparisons between different tests, we derived adjusted raw scores for age, sex and education using the Italian normative scores. Then we calculated $z$-scores, using as reference the study population. Differences in discrete and continuous variables were analyzed using the $\chi^{2}$ test, Kruskall-Wallis test and Mann-Whitney U test, with the implementation of Bonferroni's correction for multiple comparisons, respectively. A $p$ value $<0.05$ was considered significant.

Data were analyzed using IBM SPSS Statistics for Windows, version 26.0. (Armonk, NY, USA: IBM Corp.) and Graph Pad Prism 8.4.2.

\section{Results}

We collected neuropsychological evaluations from 609 ALS patients. In the whole population, $97.8 \%$ of patients were right-handed and we did not stratify the analysis according to handedness. The main descriptive statistics are summarized in Table 1, classified according to the site/side of onset (bulbar onset, $S_{\text {ri }}, S_{\text {le }}$ and $S_{\text {bil }}$ ). We also further detailed in Table $S 1$ the exact number of patients considered for each neuropsychological test.

The study population was not significantly different from PARALS population [10] (see Table S2). Compared to patients with lateralized $\left(\mathrm{S}_{\mathrm{ri}}\right.$ and $\left.\mathrm{S}_{\mathrm{le}}\right)$ spinal onset, patients with bulbar onset were older (Mann-Whitney-U test $p=0.017$ ) and predominantly female (bulbar $58.7 \%$ vs. spinal $34.5 \%$, chi-square test $p<0.001$ ), as expected, while also patients with bilateral spinal onset were more frequently older (bilateral vs. lateralized spinal onset, Mann-Whitney-U test $p=0.020$ ). Considering proportions, right-side was the most frequent side of onset in the upper limb onset, while left-side and bilateral onset were more frequent in patients with lower limb onset.

According to ALS-FTD consensus criteria [12], more than a half of bulbar onset patients were classified as cognitively impaired, with $18.8 \%$ of patients diagnosed with ALS-FTD. Considering adjusted scores for single neuropsychological tests, we found significant differences according to the site and side of onset in most of them. Bulbar onset patients were generally characterized by lower scores, usually followed by $\mathrm{S}_{\text {bil }}$ (i.e., in MMSE, FAS, CAT, FAB, RAVLT delayed recall, BSRT and CPM47). Using $z$-scores, we compared all the different tests in the whole study population. All median $z$-scores values were furtherly compared using appropriate non-parametric tests: bulbar onset patients resulted having a significantly worse cognitive impairment than spinal onset patients (Mann-Whitney $U$ test comparison among median $z$-scores, $p=0.031$, see also Figure $\mathrm{S} 1)$. Interestingly, after stratification for side of onset, patients with lateralized onset $\left(\mathrm{S}_{\mathrm{le}}\right.$ more than $\left.\mathrm{S}_{\mathrm{ri}}\right)$ obtained better scores in almost all tests, obtaining significantly different results from both bulbar and $\mathrm{S}_{\mathrm{bil}}$, which showed the same $z$-score median value (see Figure 1). 
Table 1. Descriptive statistic of amyotrophic lateral sclerosis (ALS) patients subdivided by site of onset (bulbar vs. spinal onset) and side of onset for spinal onset patients (right-side vs. left-side vs. bilateral onset).

\begin{tabular}{|c|c|c|c|c|c|c|c|}
\hline Site/Side of Onset & & Bulbar Onset & $\begin{array}{c}\text { Right-Side } \\
\text { Spinal Onset }\end{array}$ & $\begin{array}{c}\text { Left-Side } \\
\text { Spinal Onset }\end{array}$ & $\begin{array}{c}\text { Bilateral } \\
\text { Spinal Onset }\end{array}$ & Total & \\
\hline & & $n\left(\%^{r}\right)$ & $n\left(\%^{r}\right)$ & $n\left(\%^{r}\right)$ & $n\left(\%^{r}\right)$ & $n\left(\%^{r}\right)$ & \\
\hline \multirow[t]{2}{*}{ Total } & & $218(35.8)$ & $174(28.6)$ & $105(17.2)$ & $112(18.4)$ & $609(100.0)$ & \\
\hline & & $n\left(\%^{c}\right)$ & $n\left(\%^{c}\right)$ & $n\left(\%^{c}\right)$ & $n\left(\%^{c}\right)$ & $n\left(\%^{c}\right)$ & $p^{\#}$ \\
\hline Sex & & & & & & & $<0.001^{\#}$ \\
\hline Male & & $90(41.3)$ & $115(66.1)$ & $63(60.0)$ & $78(69.6)$ & $346(56.8)$ & \\
\hline Female & & $128(58.7)$ & $59(33.9)$ & $42(40.0)$ & $34(30.4)$ & $263(43.2)$ & \\
\hline Site of Onset & & & & & & & $<0.001^{\#}$ \\
\hline Bulbar Onset & & $218(100)$ & - & - & - & $218(35.8)$ & \\
\hline Upper Limbs Onset & & - & $107(61.5)$ & $45(42.9)$ & $22(19.6)$ & $174(28.6)$ & \\
\hline Lower Limbs Onset & & - & $67(38.5)$ & $60(57.1)$ & $90(80.4)$ & 217 (35.6) & \\
\hline Cognitive Classification & & & & & & & $<0.001^{\#}$ \\
\hline ALS-CN & & $102(46.8)$ & $108(62.1)$ & $68(64.8)$ & $61(54.5)$ & $339(55.7)$ & \\
\hline ALSci & & $15(6.9)$ & $23(13.2)$ & $11(10.5)$ & $11(9.8)$ & $60(9.8)$ & \\
\hline ALSbi & & $43(19.7)$ & $25(14.4)$ & $14(13.3)$ & $25(22.3)$ & $107(17.6)$ & \\
\hline ALScbi & & $17(7.8)$ & $9(5.2)$ & $6(5.7)$ & $8(7.1)$ & $40(6.6)$ & \\
\hline \multirow[t]{2}{*}{ ALS-FTD } & & $41(18.8)$ & $9(5.2)$ & $6(5.7)$ & $7(6.3)$ & $63(10.3)$ & \\
\hline & & $\begin{array}{l}\text { Median } \\
\text { (IQR) }\end{array}$ & $\begin{array}{l}\text { Median } \\
\text { (IQR) }\end{array}$ & $\begin{array}{l}\text { Median } \\
\text { (IQR) }\end{array}$ & $\begin{array}{l}\text { Median } \\
\text { (IQR) }\end{array}$ & $\begin{array}{l}\text { Median } \\
\text { (IQR) }\end{array}$ & $p$ \\
\hline Age at Onset (Years) & & $\begin{array}{c}70.0 \\
(62.7-76.0)\end{array}$ & $\begin{array}{c}67.0 \\
(58.0-74.0)\end{array}$ & $\begin{array}{c}66.0 \\
(59.0-73.0)\end{array}$ & $\begin{array}{c}70.5 \\
(61.2-75.7)\end{array}$ & $\begin{array}{c}69.0 \\
(60.0-74.0)\end{array}$ & 0.007 * \\
\hline Education (Years) & & $\begin{array}{c}8.0 \\
(5.0-11.0)\end{array}$ & $\begin{array}{c}8.0 \\
(5.0-11.0)\end{array}$ & $\begin{array}{c}8.0 \\
(5.0-11.0)\end{array}$ & $\begin{array}{c}8.0 \\
(5.0-11.0)\end{array}$ & $\begin{array}{c}8.0 \\
(5.0-12.0)\end{array}$ & 0.221 * \\
\hline Neuropsychological Test & $n$ & $\begin{array}{l}\text { Median } \\
\text { (IQR) }\end{array}$ & $\begin{array}{l}\text { Median } \\
\text { (IQR) }\end{array}$ & $\begin{array}{l}\text { Median } \\
\text { (IQR) }\end{array}$ & $\begin{array}{l}\text { Median } \\
\text { (IQR) }\end{array}$ & $\begin{array}{l}\text { Median } \\
\text { (IQR) }\end{array}$ & $p$ \\
\hline
\end{tabular}


Table 1. Cont

\begin{tabular}{|c|c|c|c|c|c|c|c|}
\hline Site/Side of Onset & & Bulbar Onset & $\begin{array}{c}\text { Right-Side } \\
\text { Spinal Onset }\end{array}$ & $\begin{array}{c}\text { Left-Side } \\
\text { Spinal Onset }\end{array}$ & $\begin{array}{c}\text { Bilateral } \\
\text { Spinal Onset }\end{array}$ & Total & \\
\hline MMSE & 603 & $\begin{array}{c}27.4 \\
(25.4-29.2) \\
\end{array}$ & $\begin{array}{c}28.0 \\
(26.5-30.0)\end{array}$ & $\begin{array}{c}28.5 \\
(27.3-30.0) \\
\end{array}$ & $\begin{array}{c}27.4 \\
(26.0-29.4) \\
\end{array}$ & $\begin{array}{c}27.8 \\
(26.3-30.0) \\
\end{array}$ & $<0.001 *$ \\
\hline Letter Fluency Test & 584 & $\begin{array}{c}25.4 \\
(19.2-33.1)\end{array}$ & $\begin{array}{c}31.3 \\
(23.5-38.6)\end{array}$ & $\begin{array}{c}31.0 \\
(23.6-39.1)\end{array}$ & $\begin{array}{c}27.4 \\
(20.9-34.8)\end{array}$ & $\begin{array}{c}28.6 \\
(21.9-35.4) \\
\end{array}$ & $<0.001 *$ \\
\hline Category fluency test & 455 & $\begin{array}{c}17.2 \\
(14.0-21.0)\end{array}$ & $\begin{array}{c}19.8 \\
(16.5-23.1)\end{array}$ & $\begin{array}{c}19.3 \\
(16.5-22.5)\end{array}$ & $\begin{array}{c}18.8 \\
(15.5-23.9)\end{array}$ & $\begin{array}{c}19.3 \\
(15.5-22.3)\end{array}$ & $0.002 *$ \\
\hline FAB & 505 & $\begin{array}{c}14.7 \\
(11.9-16.3)\end{array}$ & $\begin{array}{c}15.2 \\
(13.9-16.7)\end{array}$ & $\begin{array}{c}15.3 \\
(13.5-17.1)\end{array}$ & $\begin{array}{c}14.7 \\
(12.4-16.5)\end{array}$ & $\begin{array}{c}14.9 \\
(13.3-16.5)\end{array}$ & $0.048 *$ \\
\hline Digit Span FW & 538 & $\begin{array}{c}5.5 \\
(4.7-6.1)\end{array}$ & $\begin{array}{c}5.8 \\
(5.1-6.5)\end{array}$ & $\begin{array}{c}5.5 \\
(4.9-6.2)\end{array}$ & $\begin{array}{c}5.7 \\
(5.1-6.2) \\
\end{array}$ & $\begin{array}{c}5.5 \\
(4.9-6.3)\end{array}$ & $0.026 *$ \\
\hline Digit Span BW & 442 & $\begin{array}{c}3.7 \\
(3.3-4.3)\end{array}$ & $\begin{array}{c}4.1 \\
(3.5-4.7)\end{array}$ & $\begin{array}{c}3.8 \\
(3.5-4.3)\end{array}$ & $\begin{array}{c}3.7 \\
(3.2-4.3)\end{array}$ & $\begin{array}{c}3.9 \\
(3.4-4.4)\end{array}$ & $0.013 *$ \\
\hline TMT-A & 540 & $\begin{array}{c}43.0 \\
(28.0-76.0) \\
\end{array}$ & $\begin{array}{c}39.5 \\
(23.3-63.5)\end{array}$ & $\begin{array}{c}35.0 \\
(26.0-52.0)\end{array}$ & $\begin{array}{c}37.5 \\
(25.0-54.0)\end{array}$ & $\begin{array}{c}39.0 \\
(26.0-62.0) \\
\end{array}$ & $0.176^{*}$ \\
\hline TMT-B & 540 & $\begin{array}{c}94.0 \\
(43.0-275.0)\end{array}$ & $\begin{array}{c}82.0 \\
(35.5-144.5) \\
\end{array}$ & $\begin{array}{c}61.0 \\
(38.0-139.0) \\
\end{array}$ & $\begin{array}{c}76.5 \\
(43.3-180.0) \\
\end{array}$ & $\begin{array}{c}79.0 \\
(40.0-179.8) \\
\end{array}$ & $0.024 *$ \\
\hline TMT-B-A & 540 & $\begin{array}{c}59.5 \\
(13.3-155.0)\end{array}$ & $\begin{array}{c}41.0 \\
(10.5-90.0) \\
\end{array}$ & $\begin{array}{c}28.0 \\
(7.0-94.5)\end{array}$ & $\begin{array}{c}39.0 \\
(16.0-120.5)\end{array}$ & $\begin{array}{c}45.0 \\
(12.0-121.0)\end{array}$ & $0.013 *$ \\
\hline RAVLT-ir & 281 & $\begin{array}{c}38.0 \\
(30.1-44.1) \\
\end{array}$ & $\begin{array}{c}38.3 \\
(31.6-45.5) \\
\end{array}$ & $\begin{array}{c}41.9 \\
(34.3-46.6) \\
\end{array}$ & $\begin{array}{c}38.0 \\
(34.0-43.2 \\
\end{array}$ & $\begin{array}{c}38.7 \\
(32.8-44.5)\end{array}$ & $0.083 *$ \\
\hline RAVLT-dr & 281 & $\begin{array}{c}7.8 \\
(4.8-10.3)\end{array}$ & $\begin{array}{c}7.2 \\
(5.1-10.4)\end{array}$ & $\begin{array}{c}8.9 \\
(7.2-11.2)\end{array}$ & $\begin{array}{c}7.8 \\
(5.7-9.9) \\
\end{array}$ & $\begin{array}{c}7.8 \\
(5.6-10.2)\end{array}$ & $0.004 *$ \\
\hline BSRT-ir & 289 & $\begin{array}{c}5.5 \\
(4.2-6.6)\end{array}$ & $\begin{array}{c}5.8 \\
(4.7-6.9)\end{array}$ & $\begin{array}{c}6.4 \\
(5.2-7.8)\end{array}$ & $\begin{array}{c}5.3 \\
(4.1-6.8)\end{array}$ & $\begin{array}{c}5.7 \\
(4.5-6.8)\end{array}$ & $0.005 *$ \\
\hline BSRT-dr & 279 & $\begin{array}{c}6.0 \\
(4.5-7.2)\end{array}$ & $\begin{array}{c}6.5 \\
(4.8-7.8)\end{array}$ & $\begin{array}{c}7.3 \\
(6.1-8.0)\end{array}$ & $\begin{array}{c}6.1 \\
(4.7-7.4)\end{array}$ & $\begin{array}{c}6.4 \\
(4.9-7.6)\end{array}$ & $<0.001 *$ \\
\hline
\end{tabular}


Table 1. Cont

\begin{tabular}{|c|c|c|c|c|c|c|c|}
\hline Site/Side of Onset & & Bulbar Onset & $\begin{array}{c}\text { Right-Side } \\
\text { Spinal Onset }\end{array}$ & $\begin{array}{c}\text { Left-Side } \\
\text { Spinal Onset }\end{array}$ & $\begin{array}{c}\text { Bilateral } \\
\text { Spinal Onset }\end{array}$ & Total & \\
\hline ROCFT-copy & 426 & $\begin{array}{c}30.8 \\
(23.1-33.8)\end{array}$ & $\begin{array}{c}32.4 \\
(29.3-35.1)\end{array}$ & $\begin{array}{c}32.0 \\
(29.5-34.4)\end{array}$ & $\begin{array}{c}30.8 \\
(26.3-34.1)\end{array}$ & $\begin{array}{c}31.5 \\
(26.9-34.5)\end{array}$ & 0.005 * \\
\hline ROCFT-dr & 422 & $\begin{array}{c}11.0 \\
(7.5-15.9)\end{array}$ & $\begin{array}{c}12.0 \\
(8.8-16.6)\end{array}$ & $\begin{array}{c}12.0 \\
(7.4-15.4)\end{array}$ & $\begin{array}{c}10.6 \\
(7.0-14.3)\end{array}$ & $\begin{array}{c}11.5 \\
(7.7-15.8)\end{array}$ & 0.140 * \\
\hline СРM47 & 574 & $\begin{array}{c}27.4 \\
(23.0-31.5)\end{array}$ & $\begin{array}{c}29.5 \\
(25.2-32.1)\end{array}$ & $\begin{array}{c}29.3 \\
(26.1-31.9)\end{array}$ & $\begin{array}{c}27.5 \\
(21.7-31.5)\end{array}$ & $\begin{array}{c}28.5 \\
(24.3-31.8)\end{array}$ & 0.012 * \\
\hline WCST & 187 & $\begin{array}{c}80.0 \\
(62.8-92.8)\end{array}$ & $\begin{array}{c}85.3 \\
(58.5-98.7)\end{array}$ & $\begin{array}{c}79.4 \\
(35.1-96.5)\end{array}$ & $\begin{array}{c}78.8 \\
(43.5-94.1)\end{array}$ & $\begin{array}{c}82.8 \\
(52.8-93.9)\end{array}$ & 0.724 * \\
\hline
\end{tabular}

$\%{ }^{r}$ Raw subgroup percentage; $\%^{c}$ column subgroup percentage; ${ }^{\#}$ Chi-square test; ${ }^{*}$ Kruskal-Wallis test. $p$-Value $<0.05$ were considered as significant and written in bold. (ALS-FTD, ALS with a frontotemporal dementia; ALSbi, ALS with behavioral impairment; ALSci, ALS with cognitive impairment; ALScbi, ALS-CN, ALS with normal cognition; MMSE, mini-mental state examination; WCST, Wisconsin card sorting test; TMT-A, TMT-B, TMT B-A, trail-making Test A and B and B-A; digit span FW and BW, digit span forward and backward (digit span BW); RAVLT, Rey auditory verbal learning test; BSRT, Babcock story recall test; ROCFT, Rey-Osterrieth complex figure test; CPM47, Raven's colored progressive matrices; FAB, frontal assessment battery; ir, immediate recall; dr, delayed recall; IQR, interquartile range). 

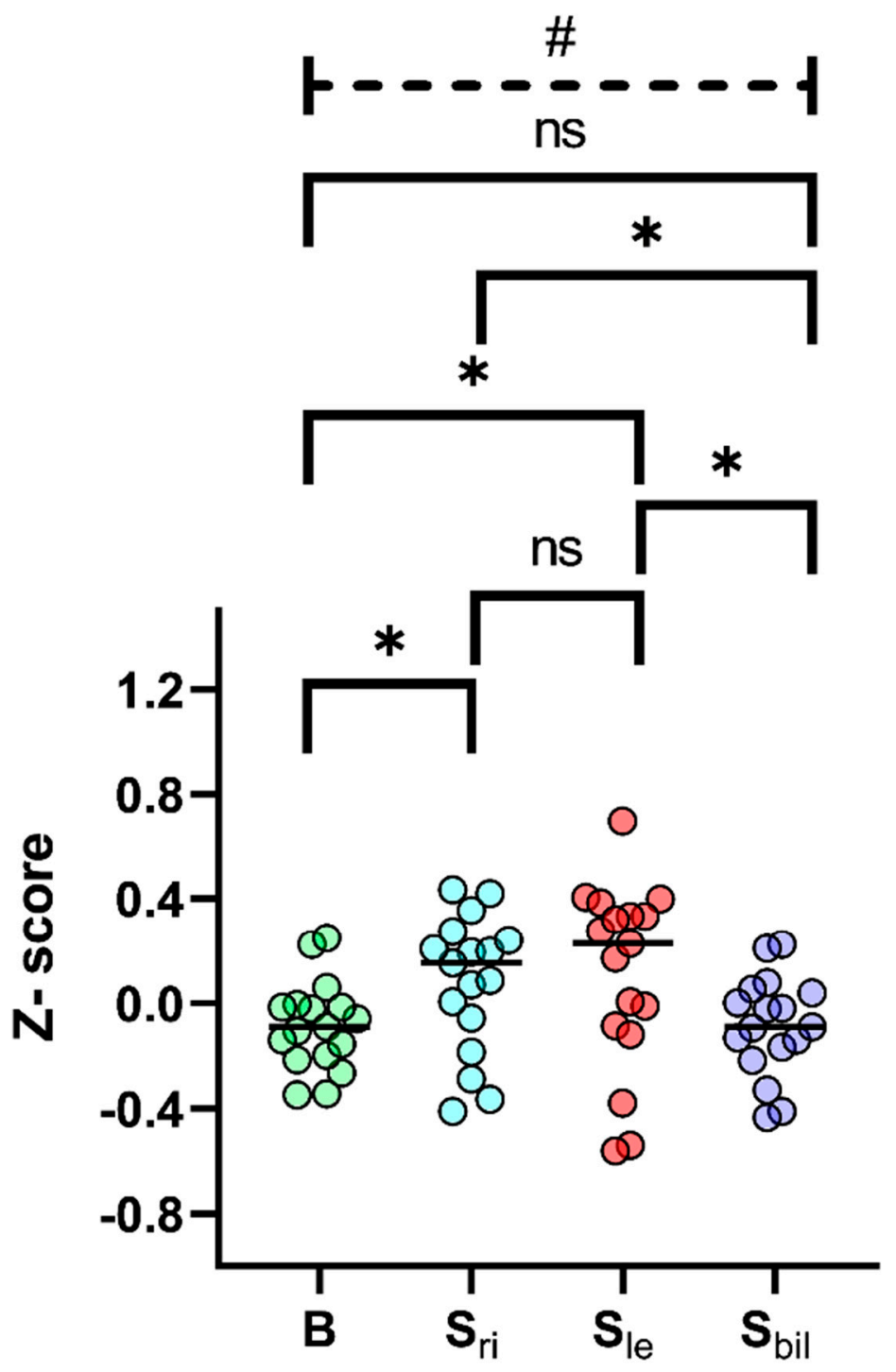

Figure 1. Median z-scores for all neuropsychological tests subdivided by site of onset (bulbar vs. spinal onset) and side of onset for spinal onset patients (right-side vs. left-side vs. bilateral onset). \# Kruskal-Wallis test $p<0.05$; * Mann-Whitney U test (with Bonferroni's correction) $p<0.05$. The black horizontal lines identified the median $z$-score for all tests. B, bulbar onset patients; $S_{\text {ri }}$, right-side spinal onset; $S_{l e}$, left-side spinal onset; $S_{b i l}$, bilateral spinal onset.

To evaluate the role of specific neuropsychological tests in determining such differences, we compared $z$-scores for each test in the overall population (see Table 2). 
Table 2. Z-score comparison according to site and side of onset (bulbar onset, B vs. right-side spinal onset, $\mathrm{S}_{\mathrm{ri}}$ vs. left-side spinal onset, $\mathrm{S}_{\mathrm{le}} \mathrm{vs}$. bilateral spinal onset,

$\left.\mathrm{S}_{\mathrm{bil}}\right)$ for different neuropsychological tests.

\begin{tabular}{|c|c|c|c|c|c|c|c|c|c|c|c|}
\hline Neuropsychological Test & Bulbar (B) & $\begin{array}{c}\text { Spinal } \\
\text { Right-Onset }\left(\mathrm{S}_{\mathrm{ri}}\right)\end{array}$ & $\begin{array}{c}\text { Spinal } \\
\text { Left-Onset }\left(\mathrm{S}_{\mathrm{le}}\right)\end{array}$ & $\begin{array}{c}\text { Spinal } \\
\text { Bil-Onset }\left(\mathrm{S}_{\mathrm{bil}}\right)\end{array}$ & $\begin{array}{l}\text { B vs. } S_{\text {ri }} \text { vs. } \\
S_{\text {le }} \text { vs. } S_{\text {bil }}\end{array}$ & B vs. $S_{\text {ri }}$ & B vs. $S_{\text {le }}$ & B vs. $S_{\text {bil }}$ & $S_{\text {ri }}$ vs. $S_{1 e}$ & $S_{\text {ri }}$ vs. $S_{\text {bil }}$ & $S_{\text {le }}$ vs. $S_{\mathrm{bil}}$ \\
\hline & $\begin{array}{l}\text { Median } \\
\text { (IQR) }\end{array}$ & Median (IQR) & Median (IQR) & Median (IQR) & $p^{\#}$ & $p^{*}$ & $p^{*}$ & $p^{*}$ & $p^{*}$ & $p^{*}$ & $p^{*}$ \\
\hline MMSE & $\begin{array}{c}-0.02 \\
(-0.78-0.66)\end{array}$ & $\begin{array}{c}0.19 \\
(-0.35-0.96)\end{array}$ & $\begin{array}{c}0.40 \\
(-0.07-0.96)\end{array}$ & $\begin{array}{c}-0.02 \\
(-0.55-0.74)\end{array}$ & $<0.001$ & 0.009 & $<0.001$ & 0.999 & 0.999 & 0.179 & 0.003 \\
\hline Letter fluency test & $\begin{array}{c}-0.35 \\
(-0.93-0.36)\end{array}$ & $\begin{array}{c}0.20 \\
(-0.53-0.88)\end{array}$ & $\begin{array}{c}0.17 \\
(-0.51-0.93)\end{array}$ & $\begin{array}{c}-0.17 \\
(-0.77-0.53)\end{array}$ & $<0.001$ & $<0.001$ & 0.001 & 0.999 & 0.999 & 0.036 & 0.053 \\
\hline Category fluency test & $\begin{array}{c}-0.34 \\
(-0.88-0.36) \\
\end{array}$ & $\begin{array}{c}0.07 \\
(-0.47-0.63) \\
\end{array}$ & $\begin{array}{c}-0.01 \\
(-0.47-0.53) \\
\end{array}$ & $\begin{array}{c}-0.10 \\
(-0.64-0.77) \\
\end{array}$ & 0.002 & 0.002 & 0.046 & 0.160 & 0.999 & 0.999 & 0.999 \\
\hline FAB & $\begin{array}{c}0.06 \\
(-0.95-0.64) \\
\end{array}$ & $\begin{array}{c}0.24 \\
(-0.23-0.78) \\
\end{array}$ & $\begin{array}{c}0.28 \\
(-0.36-0.91) \\
\end{array}$ & $\begin{array}{c}0.08 \\
(-0.76-0.69) \\
\end{array}$ & 0.048 & 0.092 & 0.479 & 0.999 & 0.999 & 0.327 & 0.916 \\
\hline Digit Span Forward & $\begin{array}{c}-0.11 \\
(-0.95-0.53)\end{array}$ & $\begin{array}{c}0.15 \\
(-0.54-0.87) \\
\end{array}$ & $\begin{array}{c}-0.09 \\
(-0.66-0.65) \\
\end{array}$ & $\begin{array}{c}0.05 \\
(-0.54-0.59) \\
\end{array}$ & 0.026 & 0.035 & 0.999 & 0.187 & 0.999 & 0.999 & 0.999 \\
\hline Digit Span Backward & $\begin{array}{c}-0.14 \\
(-0.66-0.38)\end{array}$ & $\begin{array}{c}0.21 \\
(-0.40-0.82) \\
\end{array}$ & $\begin{array}{c}-0.12 \\
(-0.41-0.43)\end{array}$ & $\begin{array}{c}-0.14 \\
(-0.78-0.45) \\
\end{array}$ & 0.013 & 0.009 & 0.999 & 0.999 & 0.405 & 0.149 & 0.999 \\
\hline TMT A & $\begin{array}{c}0.22 \\
(-0.47-0.53)\end{array}$ & $\begin{array}{c}0.29 \\
(-0.21-0.62)\end{array}$ & $\begin{array}{c}0.38 \\
(0.03-0.57)\end{array}$ & $\begin{array}{c}0.33 \\
(-0.01-0.59)\end{array}$ & 0.176 & 0.579 & 0.310 & 0.982 & 0.999 & 0.999 & 0.999 \\
\hline TMT B & $\begin{array}{c}0.26 \\
(-1.24-0.69)\end{array}$ & $\begin{array}{c}0.36 \\
(-0.16-0.75) \\
\end{array}$ & $\begin{array}{c}0.54 \\
(-0.11-0.73) \\
\end{array}$ & $\begin{array}{c}0.41 \\
(-0.45-0.69) \\
\end{array}$ & 0.024 & 0.215 & 0.029 & 0.999 & 0.999 & 0.999 & 0.734 \\
\hline TMT B-A & $\begin{array}{c}0.20 \\
(-0.91-0.73)\end{array}$ & $\begin{array}{c}0.41 \\
(-0.15-0.76)\end{array}$ & $\begin{array}{c}0.56 \\
(-0.21-0.80)\end{array}$ & $\begin{array}{c}0.43 \\
(-0.51-0.70)\end{array}$ & 0.013 & 0.083 & 0.023 & 0.999 & 0.999 & 0.999 & 0.531 \\
\hline RAVLT-ir & $\begin{array}{c}-0.09 \\
(-0.94-0.57)\end{array}$ & $\begin{array}{c}-0.06 \\
(-0.78-0.72)\end{array}$ & $\begin{array}{c}0.33 \\
(-0.50-0.84) \\
\end{array}$ & $\begin{array}{c}-0.09 \\
(-0.52-0.47)\end{array}$ & 0.083 & 0.999 & 0.112 & 0.999 & 0.480 & 0.999 & 0.178 \\
\hline RAVLT-dr & $\begin{array}{c}0.00 \\
(-0.92-0.74) \\
\end{array}$ & $\begin{array}{c}-0.19 \\
(-0.83-0.77) \\
\end{array}$ & $\begin{array}{c}0.33 \\
(-0.19-1.03) \\
\end{array}$ & $\begin{array}{c}-0.02 \\
(-0.65-0.63) \\
\end{array}$ & 0.011 & 0.999 & 0.030 & 0.999 & 0.022 & 0.999 & 0.053 \\
\hline BSRT-ir & $\begin{array}{c}-0.06 \\
(-0.67-0.50)\end{array}$ & $\begin{array}{c}0.09 \\
(-0.41-0.65) \\
\end{array}$ & $\begin{array}{c}0.41 \\
(-0.18-1.09) \\
\end{array}$ & $\begin{array}{c}-0.13 \\
(-0.71-0.59)\end{array}$ & 0.005 & 0.297 & 0.004 & 0.999 & 0.390 & 0.999 & 0.095 \\
\hline BSRT-dr & $\begin{array}{c}-0.01 \\
(-0.80-0.63)\end{array}$ & $\begin{array}{c}0.27 \\
(-0.65-0.96)\end{array}$ & $\begin{array}{c}0.70 \\
(0.08-1.07)\end{array}$ & $\begin{array}{c}0.04 \\
(-0.69-0.76)\end{array}$ & $<0.001$ & 0.790 & $<0.001$ & 0.999 & 0.033 & 0.999 & 0.006 \\
\hline ROCFT-copy & $\begin{array}{c}0.22 \\
(-0.76-0.62) \\
\end{array}$ & $\begin{array}{c}0.43 \\
(0.04-0.80) \\
\end{array}$ & $\begin{array}{c}0.39 \\
(0.06-0.70) \\
\end{array}$ & $\begin{array}{c}0.22 \\
(-0.35-0.66) \\
\end{array}$ & 0.005 & 0.007 & 0.227 & 0.999 & 0.999 & 0.129 & 0.678 \\
\hline ROCFT-dr & $\begin{array}{c}-0.15 \\
(-0.72-0.64)\end{array}$ & $\begin{array}{c}0.01 \\
(-0.52-0.74)\end{array}$ & $\begin{array}{c}0.01 \\
(-0.74-0.55)\end{array}$ & $\begin{array}{c}-0.22 \\
(-0.80-0.38)\end{array}$ & 0.140 & 0.337 & 0.999 & 0.999 & 0.999 & 0.228 & 0.999 \\
\hline
\end{tabular}


Table 2. Cont.

\begin{tabular}{|c|c|c|c|c|c|c|c|c|c|c|c|}
\hline Neuropsychological Test & Bulbar (B) & $\begin{array}{c}\text { Spinal } \\
\text { Right-Onset }\left(\mathrm{S}_{\mathrm{ri}}\right)\end{array}$ & $\begin{array}{c}\text { Spinal } \\
\text { Left-Onset }\left(\mathrm{S}_{\mathrm{le}}\right)\end{array}$ & $\begin{array}{c}\text { Spinal } \\
\text { Bil-Onset }\left(\mathrm{S}_{\mathrm{bil}}\right)\end{array}$ & $\begin{array}{l}\text { B vs. } S_{\text {ri }} \text { vs. } \\
S_{\text {le }} \text { vs. } S_{\text {bil }}\end{array}$ & B vs. $S_{\text {ri }}$ & B vs. $S_{1 \mathrm{le}}$ & B vs. $S_{\text {bil }}$ & $\mathrm{S}_{\mathrm{ri}}$ vs. $\mathrm{S}_{\mathrm{le}}$ & $S_{\text {ri }}$ vs. $S_{\text {bil }}$ & $S_{\text {le }}$ vs. $S_{\text {bil }}$ \\
\hline CPM47 & $\begin{array}{c}-0.02 \\
(-0.79-0.70)\end{array}$ & $\begin{array}{c}0.35 \\
(-0.40-0.81)\end{array}$ & $\begin{array}{c}0.32 \\
(-0.24-0.78)\end{array}$ & $\begin{array}{c}0.00 \\
(-1.03-0.71)\end{array}$ & 0.012 & 0.050 & 0.062 & 0.999 & 0.999 & 0.340 & 0.335 \\
\hline WCST TOT & $\begin{array}{c}0.25 \\
(-0.31-0.66)\end{array}$ & $\begin{array}{c}0.42 \\
(-0.45-0.85)\end{array}$ & $\begin{array}{c}0.23 \\
(-1.21-0.78)\end{array}$ & $\begin{array}{c}0.21 \\
(-0.94-0.71)\end{array}$ & 0.724 & 0.999 & 0.999 & 0.999 & 0.999 & 0.999 & 0.999 \\
\hline
\end{tabular}

" Kruskal-Wallis test. * Mann-Whitney U test with Bonferroni's correction for multiple comparisons. $p$-Value $<0.05$ were considered as significant and written in bold (MMSE, mini-mental state examination; WCST, Wisconsin card sorting test; TMT-A, TMT-B, TMT B-A, trail-making test A and B and B-A; digit span FW and BW, digit span forward and backward (digit span BW); RAVLT, Rey auditory verbal learning test; BSRT, Babcock story recall test; ROCFT, Rey-Osterrieth complex figure test; CPM47, Raven's colored progressive matrices; FAB, frontal assessment battery; ir, immediate recall; dr, delayed recall). 
A subset of tests (MMSE, letter and category fluency) obtained significantly different results among patients with bulbar onset and patients with lateralized spinal onset (both $S_{\text {ri }}$ and $S_{l e}$ ), but not among bulbar onset patients and $S_{b i l}$. Interestingly, bulbar and $S_{b i l}$ did not show any significant differences in median $z$-scores after multiple comparisons.

Digit span (both forward and backward), ROCFT copy and CPM47 obtained significantly better results in $S_{\text {ri }}$ patients compared to bulbar onset patients. Moreover, $S_{\text {ri }}$ patients had significantly better scores compared to $S_{\text {bil }}$ in letter fluency, while $S_{\text {le }}$ did not differentiate significantly.

$\mathrm{S}_{\mathrm{le}}$ were instead characterized by high $z$-scores in neuropsychological tests that evaluated verbal function, particularly verbal memory, such as RAVLT delayed recall ( $\mathrm{S}_{\mathrm{le}}$ vs. bulbar, $p=0.030$ and $\mathrm{S}_{\mathrm{le}}$ vs. $\left.\mathrm{S}_{\mathrm{ri}}, p=0.022\right)$ and BSRT immediate recall $\left(\mathrm{S}_{\mathrm{le}}\right.$ vs. bulbar, $\left.p=0.004\right)$ and delayed recall $\left(\mathrm{S}_{\mathrm{le}} \mathrm{vs}\right.$. bulbar, $p<0.001, \mathrm{~S}_{\mathrm{le}}$ vs. $\mathrm{S}_{\mathrm{ri}}, p=0.033$, and $\mathrm{S}_{\mathrm{le}}$ vs. $\left.\mathrm{S}_{\mathrm{bil}}, p=0.006\right)$. Compared to bulbar onset patients, $\mathrm{S}_{\mathrm{le}}$ showed better scores also in TMT B $(p=0.029)$ and TMT B-A $(p=0.023)$.

\section{Discussion}

Our findings point out that cognitive features of ALS patients are related to motor impairment and follow hemispheric lateralization, suggesting a possible disease spreading or simultaneous degeneration of highly interconnected frontal and precentral neurons. In keeping with previous studies, we have found that patients with bulbar onset are generally more cognitively impaired than those with spinal onset [8]. However, when we subdivided patients not only according to the site of onset (bulbar vs. spinal), but also according to the side of onset, we found that spinal patients with symmetric motor impairment have significantly worse cognitive performance than those with lateralized damage, and appear to have a cognitive dysfunction similar to bulbar patients. ALS is characterized by a focal onset, followed by a regional spreading that generally maintains a certain grade of asymmetry [15]. We used motor dysfunction as a proxy for contralateral hemispheric damage in order to evaluate the ability of neuropsychological tests in assessing cognitive functions that are highly lateralized, such as verbal memory and visuospatial abilities. Since the description of the ALS-FTD spectrum [16], the interconnection between motor and cognitive impairment has become one of the main topics in the ALS research field. Recent findings have pointed out that the extension of motor impairment, measured using King's and MiToS clinical staging systems, is correlated with cognitive decline [8]. Despite this, the complex mechanisms underlining their relationship are far from being fully understood and probably only in vivo neurodegeneration monitoring through neuroimaging could help to solve this problem [17].

The role of lateralization of motor damage in ALS has been widely studied [2,4], but, to the best of our knowledge, at present, no study has compared the initial asymmetrical features of motor impairment to specific cognitive tasks in a large cohort of ALS patients [18]. In Parkinson's disease (PD), which is another neurodegenerative disorder characterized by both motor and cognitive impairment, the asymmetry of extrapyramidal signs is included in the diagnostic criteria [19], but its underlying mechanisms have not been fully understood yet [20]. The dominant motor cortex, which is typically related to handedness, is characterized by a greater connectivity compared to the non-dominant side [21]. Moreover, transcranial magnetic stimulation studies [22] in healthy people have demonstrated a greater neuronal excitability in the dominant hemisphere, confirming a great difference in neurophysiology of the cerebral hemispheres. In ALS, handedness more than footedness has been related to the side of symptom onset and motor impairment [4], influencing, mainly for upper motor neuron features, the subsequent spread of the disease [23]. Additionally, in PD the dominant right hand is the most common site of onset [20] and when affected, it is correlated with global worse cognitive dysfunction [24]. On the contrary, left handedness and the non-dominant side of onset are associated with milder phenotypes [25]. In our ALS cohort, we only partly confirmed these observations: the subgroup $S_{l e}$, with their dominant left hemisphere less affected, showed the highest median $z$-score in the majority of neuropsychological tests, especially in the most frequently used, such as MMSE, TMT and verbal function tests. 
Considering the absence of significant lateralization of FAS and CAT, we can speculate that those tasks are not specifically influenced by left hemisphere impairment, confirming the results of a previous study in which ALS patients with verbal fluency deficits were characterized by bilateral dorsolateral prefrontal cortex, lateral and medial premotor cortex, insular cortex, and thalamus dysfunction [26].

On the contrary, we found a strong lateralization in neuropsychological tests more related to verbal memory, namely $\mathrm{RAVLT}$ and BSRT, with $\mathrm{S}_{\text {le }}$ showing significantly higher $z$-scores when compared to all other groups. $\mathrm{S}_{\mathrm{le}}$ 's left hemisphere relative preservation could justify a better performance of these subjects on verbal memory tasks. This result confirms previous diffusion-tensor imaging findings that correlate left hemisphere white matter tract integrity (specifically left perforant pathway and left uncinate fasciculus) with better performances at RAVLT and BSRT [27]. Additionally, the TMT $\mathrm{B}$ and B-A $z$-scores resulted to correlate with a left hemisphere sparing, with better performances obtained by $\mathrm{S}_{\mathrm{le}}$ when compared to bulbar patients. Our findings confirmed a recent review [28], which found evidence of an association between left-lateralized lesion sites (rostral anterior cingulate, left dorsomedial prefrontal cortex, left insular cortex, non-specific lesion sites within left prefrontal, insular, temporal and parietal cortex) and poor performance on the TMT-B.

$\mathrm{S}_{\mathrm{ri}} z$-scores for digit span backward and forward were significantly higher than bulbar patients, while $S_{\text {le }}$ and $S_{\text {bil }}$ did not differ significantly, these findings are in keeping with previous reports that correlate with both bilateral and right frontal areas (particularly right dorsolateral prefrontal cortex: DLPFC, inferior parietal lobule: IPL and anterior cingulate cortex: ACC) with short-term verbal retention tasks $[29,30]$. In $S_{\text {ri }}$, the relative sparing of right cerebral frontal areas, such as DLPFC, could explain higher performances for digit span backward and forward.

Considering ROCFT copy and CPM47, we observed a significant difference between bulbar onset and $S_{\text {ri }}$ patients. These results show that left-side and bilateral impairment are crucial in determining a worse performance in these tasks, in part confirming what is observed in PD, where a left-side predominant involvement is related to specific cognitive decline in visuospatial performances [31,32]. To our knowledge, no previous data on ROCFT and CPM47 lateralization in the ALS population were published.

This study has some limitations. First, the definition of the side of onset was based on patient clinical history; more objective data, such as the measure of strength with specific rating scale (i.e., Medical Research Council: MRC), would have improved this information. Second, our results are based on clinical and neuropsychological data, a correlation with neuroimaging data (MRI, PET) would be useful to identify the anatomical and functional correlates of our findings. Third, by considering neuropsychological evaluation at diagnosis and patients' history, we could not clarify the causality or the order of involvement of cognitive and motor dysfunction, but we only assessed their compresence.

\section{Conclusions}

We described, for the first time in ALS patients, a direct relationship between lateralized motor and cognitive features, using a properly sized cohort. Our findings are in keeping with evidence in other neurodegenerative diseases, such as PD. They could be useful in many research fields, such as neuropathology and neuroimaging, but also in clinical practice, helping to stratify patients into different cognitively susceptible categories to monitor disease progression more effectively and organize care management properly.

Supplementary Materials: The following are available online at http://www.mdpi.com/2076-3425/10/9/650/s1, Table S1: Number of patients who underwent different neuropsychological tests grouped by site/side of onset, Table S2: Comparison between PARALS population (2010-2018 period) and Turin ALS Centre who underwent neuropsychological evaluation. Figure S1: Median $z$-scores for all neuropsychological tests subdivided by site of onset (bulbar vs. spinal onset) * Mann-Whitney U test (with Bonferroni's correction) $p<0.05$.

Author Contributions: Conceptualization, U.M. and L.P.; methodology, U.M., L.P. and B.I.; software, U.M.; validation, U.M. and L.P.; formal analysis, U.M. and L.P.; investigation, U.M., L.P., B.I., A.C. (Antonio Canosa), R.V., F.P., M.C.T., L.S., M.D., M.G. and C.M.; resources, U.M., L.P., B.I., A.C. (Antonio Canosa), R.V., F.P., M.C.T., L.S., M.D., M.G. and C.M.; data curation, U.M., L.P. and B.I.; writing-original draft preparation, U.M. and L.P.; 
writing-review and editing, U.M., L.P., B.I., M.P., A.C. (Adriano Chiò) and M.C.; visualization, U.M., L.P., B.I., A.C. (Antonio Canosa), R.V., F.P., M.C.T., L.S., M.D., M.G., C.M., M.P., A.C. (Adriano Chiò) and M.C.; supervision, A.C. (Antonio Canosa), C.M., M.P., A.C. (Adriano Chiò) and M.C.; project administration, M.P., A.C. (Adriano Chiò), M.C.; funding acquisition, M.P., A.C. (Adriano Chiò), M.C. All authors have read and agreed to the published version of the manuscript.

Funding: This research received no external funding.

Conflicts of Interest: U.M., L.P., B.I., A.C. (Antonio Canosa), R.V., F.P., M.C.T., L.S., M.D., M.G., C.M., M.P. and M.C. report no conflicts of interest. A.C. (Adriano Chiò) serves on scientific advisory boards for Mitsubishi Tanabe, Roche, Biogen, and Cytokinetics. The funders had no role in the design of the study; in the collection, analyses, or interpretation of data; in the writing of the manuscript, or in the decision to publish the results.

\section{References}

1. Van Es, M.A.; Hardiman, O.; Chio, A.; Al-Chalabi, A.; Pasterkamp, R.J.; Veldink, J.H.; Van den Berg, L.H. Amyotrophic lateral sclerosis. Lancet 2017, 390, 2084-2098. [CrossRef]

2. Ravits, J.; Paul, P.; Jorg, C. Focality of upper and lower motor neuron degeneration at the clinical onset of ALS. Neurology 2007, 68, 1571-1575. [CrossRef] [PubMed]

3. Grad, L.I.; Rouleau, G.A.; Ravits, J.; Cashman, N.R. Clinical Spectrum of Amyotrophic Lateral Sclerosis (ALS). Cold Spring Harb. Perspect. Med. 2017, 7, a024117. [CrossRef] [PubMed]

4. $\quad$ Turner, M.R.; Wicks, S.; Brownstein, C.A.; Massagli, M.P.; Toronjo, M.; Talbot, K.; Al-Chalabi, A. Concordance between site of onset and limb dominance in amyotrophic lateral sclerosis. J. Neurol. Neurosurg. Psychiatry 2011, 82, 853-854. [CrossRef] [PubMed]

5. Cavallo, M.; Adenzato, M.; MacPherson, S.E.; Enrici, I.; Abrahams, S. Evidence of social understanding impairment in patients with Amyotrophic Lateral Sclerosis. PLoS ONE 2011, 6, e25948. [CrossRef] [PubMed]

6. Phukan, J.; Elamin, M.; Bede, P.; Jordan, N.; Gallagher, L.; Byrne, S.; Lynch, C.; Pender, N.; Hardiman, O. The syndrome of cognitive impairment in amyotrophic lateral sclerosis: A population-based study. J. Neurol. Neurosurg. Psychiatry 2012, 83, 102-108. [CrossRef]

7. Bersano, E.; Sarnelli, M.F.; Solara, V.; Lazzolino, B.; Peotta, L.; De Marchi, F.; Facchin, A.; Moglia, C.; Canosa, A.; Calvo, A.; et al. Decline of cognitive and behavioral functions in amyotrophic lateral sclerosis: A longitudinal study. Amyotroph. Later. Scler. Frontotemporal Degener. 2020, 1-7. [CrossRef]

8. Chiò, A.; Moglia, C.; Canosa, A.; Manera, U.; Vasta, R.; Brunetti, M.; Barberis, M.; Corrado, L.; D'Alfonso, S.; Bersano, E.; et al. Cognitive impairment across ALS clinical stages in a population-based cohort. Neurology 2019, 93, e984-e994. [CrossRef]

9. Chiò, A.; Pagani, M.; Agosta, F.; Calvo, A.; Cistaro, A.; Filippi, M. Neuroimaging in amyotrophic lateral sclerosis: Insights into structural and functional changes. Lancet Neurol. 2014, 13, 1228-1240. [CrossRef]

10. Chiò, A.; Mora, G.; Moglia, C.; Manera, U.; Canosa, A.S.; Ilardi, A.; Bertuzzo, D.; Bersano, E.; Cugnasco, P.; Grassano, M.; et al. Secular Trends of Amyotrophic Lateral Sclerosis: The Piemonte and Valle d'Aosta Register. JAMA Neurol. 2017, 74, 1097-1104. [CrossRef]

11. Rascovsky, K.; Hodges, J.R.; Knopman, D.; Mendez, M.F.; Kramer, J.H.; Neuhaus, J.; Van Swieten, J.C.; Seelaar, H.; Dopper, E.G.P.; Onyike, C.U.; et al. Sensitivity of revised diagnostic criteria for the behavioural variant of frontotemporal dementia. Brain 2011, 134, 2456-2477. [CrossRef] [PubMed]

12. Strong, M.J.; Abrahams, S.; Goldstein, L.H.; Woolley, S.; Mclaughlin, P.; Snowden, J.; Mioshi, E.; Roberts-South, A.; Benatar, M.; HortobáGyi, T.; et al. Amyotrophic lateral sclerosis: Frontotemporal spectrum disorder (ALS-FTSD): Revised diagnostic criteria. Amyotroph. Later. Scler. Frontotemporal Degener. 2017, 18, 153-174. [CrossRef] [PubMed]

13. Iazzolino, B.; Pain, D.; Peotta, L.; Calvo, A.; Moglia, C.; Canosa, A.; Manera, U.; Ilardi, A.; Bombaci, A.; Zucchetti, J.P.; et al. Validation of the revised classification of cognitive and behavioural impairment in ALS. J. Neurol. Neurosurg. Psychiatry 2019, 90, 734-739. [CrossRef] [PubMed]

14. Chiò, A.; Calvo, A.; Moglia, C.; Mazzini, L.; Mora, G.; PARALS Study Group. Phenotypic heterogeneity of amyotrophic lateral sclerosis: A population based study. J. Neurol. Neurosurg. Psychiatry 2011, 82, 740-746. [CrossRef] [PubMed]

15. Eisen, A.; Braak, H.; Del Tredici, K.; Lemon, R.; Ludolph, A.C.; Kiernan, M.C. Cortical influences drive amyotrophic lateral sclerosis. J. Neurol. Neurosurg. Psychiatry 2017, 88, 917-924. [CrossRef] [PubMed] 
16. Neary, D.; Snowden, J.S.; Mann, D.M. Cognitive change in motor neurone disease/amyotrophic lateral sclerosis (MND/ALS). J. Neurol. Sci. 2000, 180, 15-20. [CrossRef]

17. Kassubek, J.; Pagani, M. Imaging in amyotrophic lateral sclerosis: MRI and PET. Curr. Opin. Neurol. 2019, 32, 740-746. [CrossRef]

18. Tsermentseli, S.; Leigh, P.N.; Goldstein, L.H. The anatomy of cognitive impairment in amyotrophic lateral sclerosis: More than frontal lobe dysfunction. Cortex 2012, 48, 166-182. [CrossRef]

19. Postuma, R.B.; Berg, D.; Stern, M.; Poewe, W.; Olanow, C.W.; Oertel, W.; Obeso, J.; Marek, K.; Litvan, I.; Lang, A.E.; et al. MDS clinical diagnostic criteria for Parkinson's disease. Mov. Disord. 2015, 30, 1591-1601. [CrossRef]

20. Van der Hoorn, A.; Burger, H.; Leenders, K.L.; De Jong, B.M. Handedness correlates with the dominant Parkinson side: A systematic review and meta-analysis. Mov. Disord. 2012, 27, 206-210. [CrossRef]

21. Hammond, G. Correlates of human handedness in primary motor cortex: A review and hypothesis. Neurosci. Biobehav. Rev. 2002, 26, 285-292. [CrossRef]

22. Priori, A.; Oliviero, A.; Donati, E.; Callea, L.; Bertolasi, L.; Rothwell, J.C. Human handedness and asymmetry of the motor cortical silent period. Exp. Brain Res. 1999, 128, 390-396. [CrossRef] [PubMed]

23. Devine, M.S.; Kiernan, M.C.; Heggie, S.; McCombe, P.A.; Henderson, R.D. Study of motor asymmetry in ALS indicates an effect of limb dominance on onset and spread of weakness, and an important role for upper motor neurons. Amyotroph. Later. Scler. Frontotemporal Degener. 2014, 15, 481-487. [CrossRef] [PubMed]

24. Williams, L.N.; Seignourel, P.; Crucian, G.P.; Okun, M.S.; Rodriguez, R.L.; Skidmore, F.M.; Foster, P.S.; Jacobson, C.E.; Romrell, J.; Bowers, D.; et al. Laterality, region, and type of motor dysfunction correlate with cognitive impairment in Parkinson's disease. Mov. Disord. 2007, 22, 141-145. [CrossRef] [PubMed]

25. Munhoz, R.P.; Espay, A.J.; Morgante, F.; Li, J.-Y.; Teive, H.A.; Dunn, E.; Gallin, E.; Litvan, I. Long-duration Parkinson's disease: Role of lateralization of motor features. Parkinsonism Relat. Disord. 2013, 19, 77-80. [CrossRef]

26. Abrahams, S.; Leigh, P.N.; Kew, J.J.; Goldstein, L.H.; Lloyd, C.M.; Brooks, D.J. A positron emission tomography study of frontal lobe function (verbal fluency) in amyotrophic lateral sclerosis. J. Neurol. Sci. 1995, 129, 44-46. [CrossRef]

27. Christidi, F.; Karavasilis, E.; Zalonis, I.; Ferentinos, P.; Giavri, Z.; Wilde, E.A.; Xirou, S.; Rentzos, M.; Zouvelou, V.; Velonakis, G.; et al. Memory-related white matter tract integrity in amyotrophic lateral sclerosis: An advanced neuroimaging and neuropsychological study. Neurobiol. Aging 2017, 49, 69-78. [CrossRef]

28. Varjacic, A.; Mantini, D.; Demeyere, N.; Gillebert, C.R. Neural signatures of Trail Making Test performance: Evidence from lesion-mapping and neuroimaging studies. Neuropsychologia 2018, 115, 78-87. [CrossRef]

29. Gerton, B.K.; Brown, T.T.; Meyer-Lindenberg, A.; Kohn, P.; Holt, J.L.; Olsen, R.K.; Berman, K.F. Shared and distinct neurophysiological components of the digits forward and backward tasks as revealed by functional neuroimaging. Neuropsychologia 2004, 42, 1781-1787. [CrossRef]

30. Aleman, A.; Van't Wout, M. Repetitive transcranial magnetic stimulation over the right dorsolateral prefrontal cortex disrupts digit span task performance. Neuropsychobiology 2008, 57, 44-48. [CrossRef]

31. Karádi, K.; Lucza, T.; Aschermann, Z.; Komoly, S.; Deli, G.; Bosnyák, E. Visuospatial impairment in Parkinson's disease: The role of laterality. Laterality 2015, 20, 112-127. [CrossRef] [PubMed]

32. Poletti, M.; Frosini, D.; Pagni, C.; Baldacci, F.; Giuntini, M.; Mazzucchi, S.; Tognoni, G.; Lucetti, C.; Del Dotto, P.; Ceravolo, R.; et al. The relationship between motor symptom lateralization and cognitive performance in newly diagnosed drug-naïve patients with Parkinson's disease. J. Clin. Exp. Neuropsychol. 2013, 35, 124-131. [CrossRef] [PubMed]

(C) 2020 by the authors. Licensee MDPI, Basel, Switzerland. This article is an open access article distributed under the terms and conditions of the Creative Commons Attribution (CC BY) license (http://creativecommons.org/licenses/by/4.0/). 